\title{
Reduced effect of impurities on the universal pairing scale in the cuprates
}

\author{
A. B. Vorontsov, ${ }^{1}$ Ar. Abanov, ${ }^{2}$ M. G. Vavilov, ${ }^{3}$ and A. V. Chubukov ${ }^{3}$ \\ ${ }^{1}$ Department of Physics, Montana State University, Bozeman, Montana 59717, USA \\ ${ }^{2}$ Department of Physics, Texas A\&M University, College Station, Texas 77843, USA \\ ${ }^{3}$ Department of Physics, University of Wisconsin, Madison, Wisconsin 53706, USA
}

(Received 24 September 2009; revised manuscript received 13 November 2009; published 29 January 2010)

\begin{abstract}
We consider the effect of nonmagnetic impurities on the onset temperature $T^{*}$ for the $d$-wave pairing in spin-fluctuation scenario for the cuprates. We analyze intermediate coupling regime when the magnetic correlation length $\xi / a>1$ and the dimensionless coupling $u$ is $O(1)$. In the clean limit, $T^{*} \approx 0.02 v_{f} / a$ in this parameter range and weakly depends on $\xi$ and $u$. We found numerically that this universal pairing scale is also quite robust with respect to impurities: the scattering rate $\Gamma_{c r}$ needed to bring $T^{*}$ down to zero is about four times larger than in weak coupling, in good quantitative agreement with experiments. We provide analytical reasoning for this result.
\end{abstract}

DOI: 10.1103/PhysRevB.81.012508

PACS number(s): 74.20.Mn, 74.62.Dh

\section{INTRODUCTION}

The issue of the pairing glue in the cuprates is still one of the hottest topics in the physics of strongly correlated electrons. Many researchers believe that the pairing glue is a spin-fluctuation exchange, at least in the overdoped and optimally doped cuprates. At such dopings correlations are relatively strong but not enough so to break a large normal-state Fermi surface (FS) into the hole and electron pockets.

Recently, Abanov et al. ${ }^{1}$ analyzed the pairing problem in the cuprates within spin-fluctuation scenario under the assumption that the FS in the normal state is large. Within this scenario, the onset of the pairing at $T=T^{*}$ marks the development of the pseudogap phase with strong thermal fluctuations of the pairing gap, and the true superconductivity emerges at a smaller $T_{c} \leq T^{*}$, when thermal fluctuations become weaker. These authors found a smooth crossover between the limit when the interaction $U$ is smaller than $v_{f} / a$, and the pairing is confined to a vicinity of hot spots [points on the FS separated by $(\pi, \pi)$ ], and the limit of strong interaction, when the entire FS is "hot" $\left(v_{f}\right.$ is the bare Fermi velocity as obtained in band theory and $a$ is the interatomic spacing, $\left.v_{f} / a \sim 1 \mathrm{eV}\right)$. The onset temperature for the pairing, $T^{*}$, scales as $T^{*} \sim\left(v_{f} / a\right) u$ for $u=3 U a / 8 \pi v_{f} \ll 1$ and as $T^{*}$ $\sim\left(v_{f} / a\right)(1 / u)$ at large $u$, and weakly depends on $\xi$ for $\xi$ $>a$. For intermediate values of $u=O(1)$, which are mostly relevant to the cuprates, $T^{*}$ goes through a shallow maximum and is approximately $0.02 v_{f} / a$. The same pairing scale was obtained in FLEX calculations for the Hubbard model, ${ }^{2}$ in two-particle self-consistent calculations, ${ }^{3}$ in dynamical cluster approximation, ${ }^{4}$ and in cluster DMFT. ${ }^{5}$ The good agreement between all these results is strong indication that $T^{*} \sim 0.02 v_{f} / a \sim 200-250 \mathrm{~K}$ is indeed the universal pairing scale in optimally doped cuprates. At smaller dopings, this scenario breaks down because of electron localization which gives rise to precursors to hole and electron FS pockets already in the normal state. The pseudogap temperature $T^{*}$ then becomes a scale at which the system develops such precursors while the pairing emerges at a smaller temperature due to interaction between electron pockets.

The subject of this communication is the analysis of how the universal pairing scale $T^{*}$ in optimally doped cuprates is affected by nonmagnetic impurities, which are pair breaking for unconventional superconductors. In near-optimally doped cuprates, concentrations of dopants are quite substantial, and potential random scattering off dopants could significantly reduce $T^{*}$. At weak coupling, which in our case corresponds to small $u$ and $\xi \sim a$, nonmagnetic impurities in a $d$-wave superconductor suppress $T^{*}$ in the same way as magnetic impurities in a BCS superconductor and $T^{*}$ is given by Abrikosov-Gorkov (AG) formula ${ }^{6} \log T_{0}^{*} / T^{*}=\Psi(1 / 2$ $\left.+\Gamma / 2 \pi T^{*}\right)-\Psi(1 / 2)$, where $\Psi(x)$ is the di-Gamma function and $T_{0}^{*}$ is the pairing temperature in the absence of impurities (for an $s$-wave and magnetic impurities, the formula is the same, ${ }^{7}$ but with $\Gamma / 2$ instead of $\Gamma$ ). The ratio of the critical value of the scattering to $T_{0}^{*}$ is $\Gamma_{c r} / T_{0}^{*}=\pi / 2 e^{0.5772} \approx 0.88$ $\left(T^{*}\left(\Gamma_{c r}\right)=0\right)$.

The issue we address here both analytically and numerically is what is this ratio when $u=O(1)$ and $\xi \gg a$, when the pairing problem involves incoherent fermions and neargapless dynamical bosons and is very different from the $d$-wave version of the BCS theory. We find that in this situation the ratio $\Gamma_{c r} / T_{0}^{*}$ is about four times larger than 0.88 , i.e., the pairing is much less suppressed by impurities than in the weak coupling. This result is in agreement with the experiments which observed ${ }^{8}$ a similar reduction in the slope of $T^{*}(\Gamma)$ compared with the AG formula.

Another issue that we consider here is how impurity scattering affects the angular dependence of the $d$-wave pairing gap. For a clean system, Abanov et al. ${ }^{1}$ have found that in the universal regime the form of the gap $\Delta_{p}(\omega)$ is very close to $\cos p_{x}-\cos p_{y}$ for all frequencies. We show that the $\cos p_{x}$ $-\cos p_{y}$ form holds in the presence of impurities-the angular dependence only slightly changes with $\Gamma$. The implication is that both $T^{*}$ and the gap structure are robust toward impurities.

The angular dependence of the gap, particularly in underdoped cuprates, has been the subject of intensive debates recently and some ARPES data were interpreted as evidence for strong deviations from the $\cos p_{x}-\cos p_{y}$ form. We emphasize in this regard that the position of the maximum of the spectral function $A_{p}(\omega)$ represents the pairing gap $\Delta_{p}(\omega)$ only deep in the superconducting state. At higher $T$, the po- 
sition of the maximum in $A_{p}(\omega)$ differs from $\Delta_{p}(\omega)$ because of damping induced by scattering off thermal bosons. In particular, even for a gap with a perfect $\cos p_{x}-\cos p_{y}$ form a maximum in $A_{p}(\omega=0)$ is still present in some neighborhood of a node (a Fermi arc). In this regard, our result that the gap keeps $\cos p_{x}-\cos p_{y}$ form even in the presence of impurities agrees with ARPES data by Campuzano and co-workers, ${ }^{9}$ who detected this form at the lowest $T$.

The effects of nonmagnetic and magnetic impurities in superconductors with unconventional order parameters have been studied for high- $T_{c}$ cuprates, ${ }^{10-15}$ noncuprate superconductors, ${ }^{16-19}$ and most recently for the pnictides. ${ }^{20,21}$ For the cuprates, most of the studies attributed a slow decrease in $T^{*}$ to the extended nature of the impurity potential $^{12-14}$ but Monthoux and Pines ${ }^{10}$ performed a numerical analysis of $T^{*}$ suppression in YBCO by nonmagnetic Ni impurities and found that the initial slope of $T^{*}$ is quite small even when impurities are pointlike scatterers. Very recently, Kemper et al. ${ }^{15}$ studied the effect of disorder using dynamical cluster approximation and quantum Monte Carlo, and found that ordinary pair breaking by impurities is partly balanced by the impurity-induced enhancement of spin correlations which increases the pairing interaction mediated by spin fluctuations

Our result agree with Kemper et al. ${ }^{15}$ and also Graser et $a l .{ }^{14}$ in that the origin of the flattening of $T^{*}(\Gamma)$ are magnetic strong-correlation effects. At the same time, we found that, in the universal regime, $T^{*}$ very weakly depends on the spin-correlation length $\xi$. In our theory, softness of $T^{*}$ suppression compared to weak-coupling AG theory is primarily associated with the strong frequency dependence of the pairing interaction.

\section{THEORY}

We follow earlier work ${ }^{1}$ and consider fermions with a large FS and $d$-wave pairing mediated by overdamped spin fluctuations. We add to earlier analysis an isotropic, elastic scattering by pointlike impurities. As customary for the pairing problem, we introduce normal and anomalous Green's functions and self-energies and treat spin-fluctuation mediated pairing within the Eliashberg theory, by keeping selfenergies but neglecting vertex corrections. For small and large $u$ 's, this approximation can be rigorously justified because vertex corrections are small in $u$ or $1 / u$, respectively. For $u=O(1)$, it can only be justified on the basis that vertex corrections are small numerically. ${ }^{22}$

The set of equations includes fermionic and bosonic selfenergies in the normal state and the linearized equation for the $d$-wave pairing vertex $\Phi_{\boldsymbol{p}_{f}}^{\chi}\left(\omega_{m}\right)$ (Ref. 1)

$$
\begin{gathered}
\Sigma_{\boldsymbol{p}_{f}}\left(\omega_{m}\right)=\pi T^{*} \sum_{\omega_{m}^{\prime}} \int d \boldsymbol{p}_{f}^{\prime} \chi_{\boldsymbol{p}_{f}-\boldsymbol{p}_{f}^{\prime}}^{\omega_{m}-\omega_{m}^{\prime}} \operatorname{sign}\left(\omega_{m} \omega_{m}^{\prime}\right), \\
\Phi_{\boldsymbol{p}_{f}}^{\chi}\left(\omega_{m}\right)=-\pi T^{*} \sum_{\omega_{m}^{\prime}} \int d \boldsymbol{p}_{f}^{\prime} \frac{\chi_{\boldsymbol{p}_{f}-\boldsymbol{p}_{f}^{\prime}}^{\omega_{m}-\omega_{m}^{\prime}} \Phi_{\boldsymbol{p}_{f}^{\prime}}\left(\omega_{m}^{\prime}\right)}{\left|\omega_{m}^{\prime}\right|+\Gamma+\Sigma_{\boldsymbol{p}_{f}^{\prime}}\left(\omega_{m}^{\prime}\right)},
\end{gathered}
$$

$$
\begin{gathered}
\Phi_{\boldsymbol{p}_{f}}\left(\omega_{m}\right)=\Phi_{\boldsymbol{p}_{f}}^{\chi}\left(\omega_{m}\right)+\Gamma \int \frac{d \boldsymbol{p}_{f}^{\prime} \Phi_{\boldsymbol{p}_{f}^{\prime}}\left(\omega_{m}\right)}{\left|\omega_{m}\right|+\Gamma+\Sigma_{\boldsymbol{p}_{f}^{\prime}}\left(\omega_{m}\right)}, \\
\chi_{\boldsymbol{p}_{f}-\boldsymbol{p}_{f}^{\prime}}^{\Delta \omega}=\frac{(u a / \pi)}{(a / \xi)^{2}+a^{2}\left|\boldsymbol{p}_{f}-\boldsymbol{p}_{f}^{\prime}-\boldsymbol{Q}\right|^{2}+|\Delta \omega| / \Omega},
\end{gathered}
$$

where $\Omega=3 v_{f} /(16 u a)$ and momenta $\boldsymbol{p}_{f}$ in all formulas are confined to the FS because integration in the direction transverse to the FS has been carried out. In distinction to Ref. 1 in Eqs. (2) and (3), we also included impurity renormalization of $\Phi_{p_{f}}^{\chi}$ and of Matsubara energies $\omega_{m}=\pi T^{*}(2 m+1)$, where $\Gamma=\left(n_{i} / \pi N_{f}\right) \sin ^{2} \delta$ depends on impurity concentration $n_{i}$, fermionic density of states $N_{f}$, and the impurity potential $u_{0}$ via $\tan \delta=\pi u_{0} N_{f}$.

The set of equations for $\Phi$ and $\Sigma$ can be simplified in the usual way by introducing mass-renormalization factor $Z_{p_{f}}\left(\omega_{m}\right)$ and the pairing gap $\Delta_{p_{f}}\left(\omega_{m}\right)$ via

$$
Z_{\boldsymbol{p}_{f}}\left(\omega_{m}\right)=\frac{\left|\omega_{m}\right|+\Gamma+\Sigma_{\boldsymbol{p}_{f}}\left(\omega_{m}\right)}{\left|\omega_{m}\right|}, \quad \Delta_{\boldsymbol{p}_{f}}\left(\omega_{m}\right)=\frac{\Phi_{\boldsymbol{p}_{f}}\left(\omega_{m}\right)}{Z_{\boldsymbol{p}_{f}}\left(\omega_{m}\right)} .
$$

Due to $A_{1 g}$ symmetry of $\Sigma_{\boldsymbol{p}_{f}}$ and $B_{1 g}$ symmetry of $\Phi_{\boldsymbol{p}_{f}}^{\chi}$ the impurity renormalization of the pairing vertex vanishes, i.e., $\Phi_{\boldsymbol{p}_{f}}=\Phi_{\boldsymbol{p}_{f}}^{\chi}$. Using Eq. (5) we obtain from Eq. (2)

$\sum_{\omega_{m}^{\prime}} \int d \boldsymbol{p}_{f}^{\prime}\left[\pi T \frac{\chi_{\boldsymbol{p}_{f}-\boldsymbol{p}_{f}^{\prime}}^{\omega_{m}-\omega_{m}^{\prime}}}{\left|\omega_{m}^{\prime}\right|\left|\omega_{m}\right|}+\delta_{m m^{\prime}} \delta_{\boldsymbol{p}_{f} \boldsymbol{p}_{f}^{\prime}} \frac{Z\left(\omega_{m}, \boldsymbol{p}_{f}\right)}{\left|\omega_{m}\right|}\right] \Delta\left(\omega_{m}^{\prime}, \boldsymbol{p}_{f}^{\prime}\right)=0$.

We wrote the gap equation as an eigenvalue problem by moving all terms to one side and symmetrizing the kernel with respect to $\omega_{m}, \omega_{m^{\prime}}$.

\section{NUMERICAL SOLUTION}

This linearized gap equation is solved numerically by presenting the FS integral as a sum, varying $T$ and finding $T^{*}$ as the highest temperature where Eq. (6) is satisfied. The result is presented in Fig. 1. We clearly see a strong increase in the ratio $\Gamma_{c r} / T_{0}^{*}$ compared with a BCS $d$-wave superconductor. For $u \sim 1$, when $T_{0}^{*}$ as a function of $u$ has a maximum at about $0.02 v_{f} / a$, this ratio is nearly four times larger than in the BCS limit. This result is in a good quantitative agreement with the experiment in Ref. 8 and shows that the universal pairing scale in the cuprates is resistant to ordinary impurities.

In Fig. 2 on the left we show the angular dependences of the quasiparticle renormalization factor $Z_{p_{f}}\left(\omega_{0}\right)=1$ $+\left[\Sigma_{p_{f}}\left(\omega_{0}\right)+\Gamma\right] / \omega_{0}$ and of the gap function $\Delta_{p_{f}}\left(\omega_{0}\right)$ for clean and dirty cases, for $\omega_{0}=\pi T^{*}$ and different $u$ and $\xi$. In the clean case and $u=O(1)$ the angular dependence is quite close to $\cos 2 \varphi$ (or $\cos p_{x}-\cos p_{y}$ ). We see that the effect of the impurities on the angular dependence of the gap is quite small, i.e., $\cos 2 \varphi$ form is preserved in a dirty case. We verified that this holds for all $\Gamma$ up to the critical value and for all 


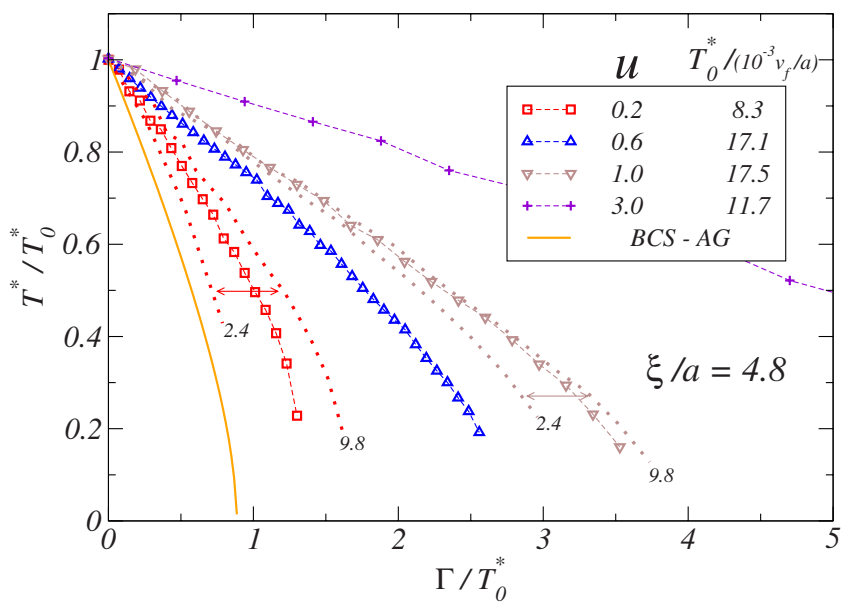

FIG. 1. (Color online) The onset temperature for the pairing, $T^{*}$, vs $\Gamma$ for different values of $u . T_{0}^{*}$ is the pairing temperature in the clean limit. $v_{f} / a \sim 1 \mathrm{eV}$ in the cuprates, hence $10^{-3} v_{f} / a \simeq 1 \mathrm{meV}$. We set $\xi / a=4.8$ and show examples of $\xi$-dependent spread from $\xi / a=2.4$ to 9.8 for $u=0.2$ and 1.0 , by dotted lines. The solid line is AG-type result for a BCS $d$-wave superconductor, which in our case corresponds to the limit $u \xi \ll 1$. The key result in this figure is a progressive increase with $u$ of the critical ratio $\Gamma_{c r} / T_{0}^{*}$, at which $T^{*}=0$. For $u=O(1)$, this ratio is about four times larger than in the BCS limit. The transition temperature is found with relative precision $10^{-2}$. The range of low $T$ requires special care because for any finite number of Matsubara points the curve $T^{*}(\Gamma)$ bends back toward the origin producing a spurious second solution.

Matsubara frequencies. For completeness, in Fig. 2(c) we show the frequency dependences of $Z_{p_{f}}\left(\omega_{m}\right)$ and $\Delta_{p_{f}}\left(\omega_{m}\right)$, and in panel (d) we present $\Phi_{p_{f}}\left(\omega_{m}\right)$ at $\varphi=0$ which we will later compare with the analytical formula.

\section{ANALYTICAL REASONING}

To understand the origin of the increase in $\Gamma_{c r} / T_{0}^{*}$ we analyze the equation for the pairing vertex $\Phi_{p_{f}}\left(\omega_{m}\right)$ analytically at $T=0$, i.e., we look for a solution near $\Gamma_{c r}$. To do this, we make an approximation and neglect angular dependence of the gap near a hot spot. The momentum integration along the FS then can be carried out analytically and the equation for the gap at a hot spot becomes one-dimensional integral equation in frequency only. This equation is more easy to analyze than the original three-dimensional (3D) integral equation. The approximation of the gap function by a constant near hot spots can be rigorously justified at small $u$ (corrections are higher powers of $u$ ) but remains qualitatively valid up to $u=O(1)$ (Ref. 1). The expression for $T_{0}^{*}$ in this approximation has been obtained earlier ${ }^{23}-T_{0}^{*}$ $\approx 0.13 u\left(v_{f} / a\right)$, with very weak dependence on $\xi$ as long as $u \xi>1$. For small $u \xi, T_{0}^{*}$ is described by a BCS formula.

Using $\Phi_{p_{f}+\boldsymbol{Q}}\left(\omega_{m}\right)=-\Phi_{p_{f}}\left(\omega_{m}\right)$, dropping the dependence of $\boldsymbol{p}_{f}$ near a hot spot, integrating over momentum in Eqs. (1)-(3) and rescaling variables, we obtain after some algebra the equation for $\Phi\left(\omega_{m}\right)$ at $T=0$ in the form
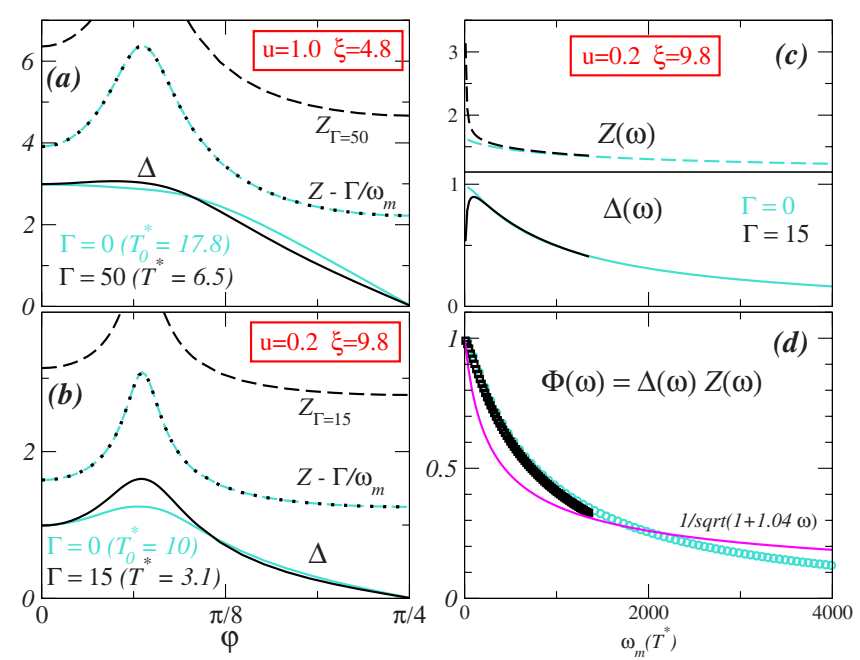

FIG. 2. (Color online) Left: the angular dependence of the quasiparticle renormalization factor $Z_{p_{f}}\left(\omega_{m=0}\right)$ and of the gap function $\Delta_{p_{f}}\left(\omega_{0}\right)$ for (a) $u=1$ and $\xi=4.8 a$ and (b) $u=0.2$ and $\xi=9.8 a . T^{*}, \Gamma$, and $\omega_{m}$ are in units of $10^{-3} v_{f} / a$. Observe that the angular dependences of the gap and $Z$ (with subtracted constant shift due to $\Gamma$ ) changes very little between $\Gamma=0$ (light lines) and $\Gamma \leqq \Gamma_{c r}$ (dark lines). Panel (c): frequency dependences of $\varphi=0 \quad \Delta_{p_{f}}\left(\omega_{m}\right)$ and $Z_{p_{f}}\left(\omega_{m}\right)$. Panel $(\mathrm{d})$ : the comparison of the numerically obtained frequency dependence of $\Phi_{\varphi=0}\left(\omega_{m}\right)$ with the analytic solution of Eq. (7) in which momentum dependence of the gap near hot spot is neglected.

$$
\begin{aligned}
\Phi(x)= & \frac{\lambda}{2} \int_{0}^{\infty} d y \frac{\Phi(y)}{y+\widetilde{\Gamma}+\frac{2 \lambda y}{1+\sqrt{4 \lambda^{2} y+1}}} \\
& \times\left(\frac{1}{\sqrt{4 \lambda^{2}|y-x|+1}}+\frac{1}{\sqrt{4 \lambda^{2}|y+x|+1}}\right),
\end{aligned}
$$

where $x, y$ are frequencies in units of $\bar{\omega}=(3 u / 4) v_{f} / a, \tilde{\Gamma}$ $=\Gamma_{c r} / \bar{\omega}$, and $\lambda=(2 u \xi)$ (mass renormalization in the normal state is $1+\lambda$ ).

Weak coupling BCS limit corresponds to $\lambda \ll 1$. In this limit, $\Phi(x)$ becomes independent of $x$ (and $\Phi=\Delta$ ), the gap equation is solved in the same way as in AG theory and the value of $\Gamma_{c r} / T_{0}^{*} \approx 0.88$. We, however, are interested in the opposite limit, when $\lambda>1$. We analyzed Eq. (7) by normalizing $\Phi(x)$ to $\Phi(0)=1$, expanding at small $x$ and at large $x$ and extrapolating between the two limits. At small $x, \Phi=1$ $-O(x)$, at large $x, \Phi(x) \propto 1 / \sqrt{x}$. We found that $\Phi(x)$ is well approximated by $\Phi(x)=1 / \sqrt{1+c x}$, where $c$ is a constant which depends on $\lambda$ and $\widetilde{\Gamma}$. The error is less than $2 \%$ for all $x$ and for all $\lambda$ which we considered. We also checked that this solution is not an artifact: if we use this $\Phi(x)$ as an input and run iterations, $\Phi(x)$ rapidly converges. The critical value of $\widetilde{\Gamma}$ is then obtained by substituting this form back into Eq. (7) and solving for $\Phi(0)=1$.

Carrying out this procedure, we found that $c$ increases with increasing $\lambda=u \xi$, i.e., when the correlation length increases and the pairing problem becomes more and more non-BCS, the gap function gets confined to progressively 
smaller frequencies. The $c(\lambda)$ increases from $c(1)=0.66$, through $c(2)=1.04$ and $c(5)=1.7$, to $c(\infty)=2.31$. In Fig. 2(d) we compare our approximate analytic $\Phi\left(\omega_{m}\right)$ for $u=0.2$, $\xi$ $=9.8 a(\lambda \approx 2)$ with the numerical $\Phi_{p_{f}}\left(\omega_{m}\right)$. We see that the agreement is expectedly not prefect but generic trends of the frequency dependence is captured by the approximate solution.

Substituting $\Phi(x)=1 / \sqrt{1+c x}$ back into Eq. (7) and solving for $\Phi(0)=1$, we found that $\widetilde{\Gamma}$ progressively increases as $\lambda$ gets larger, from $\lambda^{-2} e^{-1 / \lambda}$ at small $\lambda$ to 0.3 for $\lambda=1$ and to 0.46 for $\lambda=\infty$.

The monotonic increase in the value of $\widetilde{\Gamma}$ with increasing $\lambda$ is a tricky effect. One could expect that the confinement of $\Phi(x)$ to smaller $x$ as $\lambda$ increases and the increase in the self-energy tend to reduce $\widetilde{\Gamma}$ simply because typical frequencies get smaller. However, as $\lambda$ increases, the interaction strength also increases and this tends to increase $\widetilde{\Gamma}$ because $\widetilde{\Gamma}$ appears in the denominator in the integral for $\Phi(0)=1$, and larger $\widetilde{\Gamma}$ are required to balance the increase in the interaction. We compared the two effects and found that increase in the interaction overshadows other effects and is the origin of the growth of $\widetilde{\Gamma}$ with increasing $\lambda$.

We next compared the growth of $\Gamma_{c r}=\bar{\omega} \tilde{\Gamma}(\lambda)$ and the grown of $T_{0}^{*}$. The latter also scales as $\bar{\omega}$ with $\lambda$-dependent prefactor (Ref. 23). This prefactor increases with increasing $\lambda$ but its $\lambda$ dependence is very weak: it changes by less than $5 \%$ between $\lambda=1$ and $\lambda=\infty$. As a result, the $\lambda$ dependence of the ratio $\Gamma_{c r} / T_{0}^{*}$ predominantly comes from $\tilde{\Gamma}(\lambda)$, which, we remind, increases with $\lambda$. Inserting the numbers, we find that the ratio $\Gamma_{c r} / T_{0}^{*}$ becomes 2.0 for $\lambda=1,2.37$ for $\lambda=2,2.47$ for $\lambda=5$, and 2.74 for $\lambda=\infty$. The scale of the increase is quite consistent with what we found numerically in Fig. 1 by solving the full 3D integral equation in momentum and frequency.

\section{SUMMARY}

In this Brief Report we considered the effect of nonmagnetic impurities on the onset temperature $T^{*}$ for the $d$-wave pairing in spin-fluctuation scenario for the cuprates. Nonmagnetic impurities are pair breaking for $d$-wave superconductivity and one should expect a reduction in $T^{*}$ due to impurities. In weak coupling, $T^{*}$ falls off rapidly, following the AG curve.

We analyzed the effect of impurities in the intermediate coupling regime when the magnetic correlation length $\xi / a$ $>1$, the dimensionless coupling $u$ is $O(1)$, and the pairing problem is qualitatively different from BCS. In the clean limit, $T^{*}$ in this parameter range weakly depends on $\xi$ and $u$ and is approximately $0.02 v_{f} / a$. We found that this universal pairing scale is quite robust with respect to impurities: the critical value of the scattering rate $\Gamma_{c r}$ needed to bring $T^{*}$ down to zero is about four times larger than in the weak coupling. This implies that the slope of the initial reduction in $T^{*}$ is weaker by about the same factor than in the weak coupling. This reduction in the slope agrees with the experiments $^{8}$ and with earlier work by Monthoux and Pines ${ }^{10}$ on $T^{*}$ suppression in YBCO due to nonmagnetic Ni impurities. We also analyzed the angular dependence of the gap and found that it is little affected by impurities.

\section{ACKNOWLEDGMENTS}

We thank I. Vekhter for useful discussions. A.A. is supported by NSF under Grant No. 0757992 and Welch Foundation (Grant No. A-1678), and A.V.C. is supported by NSF under Grant No. DMR-0906953. Three of us (A.B.V., M.G.V., and A.V.C.) are thankful to the Aspen Center for Physics for hospitality.
${ }^{1}$ Ar. Abanov, A. V. Chubukov, and M. R. Norman, Phys. Rev. B 78, 220507(R) (2008).

${ }^{2}$ P. Monthoux and D. J. Scalapino, Phys. Rev. Lett. 72, 1874 (1994); St. Lenck, J. P. Carbotte, and R. C. Dynes, Phys. Rev. B 50, 10149 (1994); T. Dahm and L. Tewordt, ibid. 52, 1297 (1995); D. Manske, I. Eremin, and K. H. Bennemann, ibid. 67, 134520 (2003).

${ }^{3}$ B. Kyung, J.-S. Landry, and A.-M. S. Tremblay, Phys. Rev. B 68, 174502 (2003).

${ }^{4}$ T. A. Maier, M. Jarrell, T. C. Schulthess, P. R. C. Kent, and J. B. White, Phys. Rev. Lett. 95, 237001 (2005).

${ }^{5}$ K. Haule and G. Kotliar, Phys. Rev. B 76, 104509 (2007).

${ }^{6}$ A. A. Abrikosov and L. P. Gorkov, Sov. Phys. JETP 12, 1243 (1961).

${ }^{7}$ A. Balatsky, I. Vekhter, and J.-X. Zhu, Rev. Mod. Phys. 78, 373 (2006).

${ }^{8}$ S. K. Tolpygo, J. Y. Lin, M. Gurvitch, S. Y. Hou, and J. M. Phillips, Phys. Rev. B 53, 12454 (1996).

${ }^{9}$ M. Shi et al., EPL, 88, 27008 (2009), and references therein; A. Kanigel et al., Phys. Rev. Lett. 101, 137002 (2008); A. Kanigel, U. Chatterjee, M. Randeria, M. R. Norman, S. Souma, M. Shi, Z. Z. Li, H. Raffy, and J. C. Campuzano, ibid. 99, 157001
(2007).

${ }^{10}$ P. Monthoux and D. Pines, Phys. Rev. B 49, 4261 (1994).

${ }^{11}$ M. Franz et al., Phys. Rev. B 56, 7882 (1997).

${ }^{12}$ G. Harań and A. D. S. Nagi, Phys. Rev. B 58, 12441 (1998), and references therein.

${ }^{13}$ M. L. Kulić and O. V. Dolgov, Phys. Rev. B 60, 13062 (1999).

${ }^{14}$ S. Graser, P. J. Hirschfeld, L. Y. Zhu, and T. Dahm, Phys. Rev. B 76, 054516 (2007).

${ }^{15}$ A. Kemper, D. G. S. P. Doluweera, T. A. Maier, M. Jarrell, P. J. Hirschfeld, and H. P. Cheng, Phys. Rev. B 79, 104502 (2009).

${ }^{16}$ E. Müller-Hartmann and J. Zittartz, Phys. Rev. Lett. 26, 428 (1971).

${ }^{17}$ S. Yoksan and A. D. S. Nagi, Phys. Rev. B 30, 2659 (1984).

${ }^{18}$ G. Preosti and P. Muzikar, Phys. Rev. B 54, 3489 (1996).

${ }^{19}$ A. A. Golubov and I. I. Mazin, Phys. Rev. B 55, 15146 (1997).

${ }^{20}$ A. B. Vorontsov, M. G. Vavilov, and A. V. Chubukov, Phys. Rev. B 79, 140507(R) (2009).

${ }^{21}$ D. Parker et al., Phys. Rev. B 78, 134524 (2008).

${ }^{22}$ A. Abanov et al., Adv. Phys. 52, 119 (2003).

${ }^{23}$ Ar. Abanov, A. V. Chubukov, and A. M. Finkelstein, Europhys. Lett. 54, 488 (2001). 